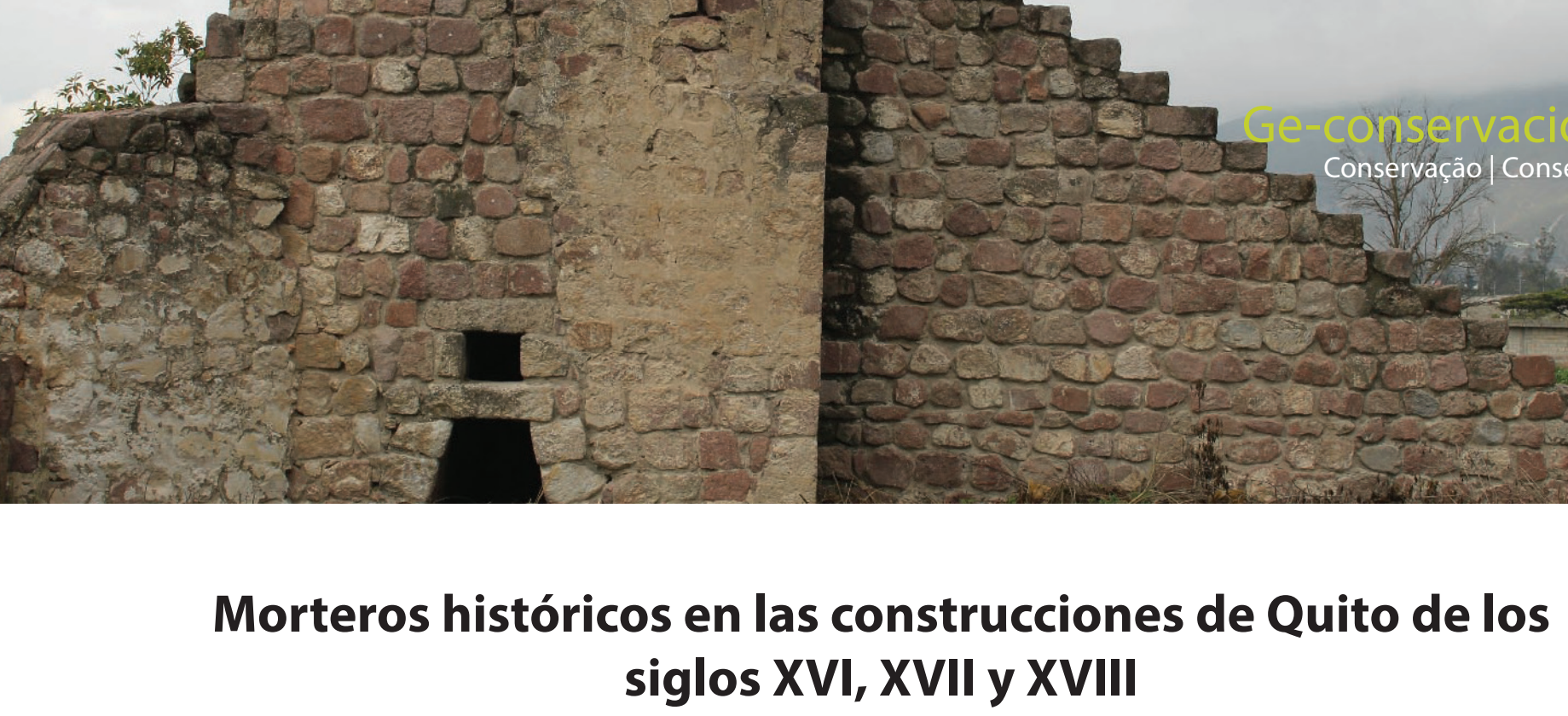

\title{
Morteros históricos en las construcciones de Quito de los siglos XVI, XVII y XVIII
}

\author{
M. Lenin Lara Calderón, David Sanz Arauz e Inés del Pino Martínez
}

\begin{abstract}
Resumen: Este artículo tiene como objetivo profundizar en la caracterización de los morteros de revestimiento en la arquitectura colonial de Quito de los siglos XVI, XVII y XVIII. Se parte del supuesto que durante la colonización hubo aportes locales y europeos en la construcción de la arquitectura religiosa de esta ciudad, los resultados que hoy están a la vista son testimonios de este proceso. El análisis de las características compositivas de los morteros, cuyas muestras provienen de los edificios que hoy conocemos, contribuyen a perfeccionar de alguna manera el rompecabezas de la historia, todavía incompleta, ya que las destrucciones producidas por terremotos, y sobre todo intervenciones de restauración o ampliación edilicia han dejado sus huellas en estos edificios.
\end{abstract}

Palabras clave: morteros de revestimiento, Quito, construcción vernácula, conventos católicos, historia de los morteros

\section{Historic mortars in the constructions of Quito of the 16th, 17th and 18th centuries}

Abstract:The objective of this article is to deepen the characterization of the coating mortars in the colonial architecture of Quito in the 16th, 17th and 18th centuries. It is assumed that during the colonization there were local and European contributions to the construction of the religious architecture of this city, the results that are now in sight are testimonies of this process. The analysis of the compositional characteristics of the mortars, whose samples come from the buildings that we know today, contribute to somehow perfect the puzzle of history, still incomplete, since the destruction caused by earthquakes, and especially restoration or building enlargement have left their traces on these buildings.

Keyword: Coating mortars, Quito, vernacular construction, catholic convents, history of the mortars

\section{Argamassas históricas dos edifícios de Quito dos séculos XVI, XVII e XVIII}

Resumo: O objetivo deste artigo é aprofundar a caracterização das argamassas de revestimento na arquitetura colonial de Quito nos séculos XVI, XVII e XVIII. Considera-se que durante a colonização houve contribuições locais e europeias para a construção da arquitetura religiosa desta cidade, como testemunham os resultados que estão à vista. A análise das características composicionais das argamassas, cujas amostras provêm dos edifícios que conhecemos hoje, contribui de alguma forma para aprofundar o conhecimento da história, que continua incompleta devido às marcas deixadas por terremotos e, principalmente, o restauro e a ampliação desses edifícios.

Palavras-chave: argamassas de revestimento, Quito, construção vernacular, conventos católicos, história das argamassas

\section{Introducción}

El homo faber expresión latina que significa el "hombre que construye", expresa la necesidad ancestral del ser humano por abrigarse para soportar las condiciones agrestes de la naturaleza; genera la necesidad de un espacio simple y habitable donde la materialidad de su entorno inmediato determinó algunas características específicas para representar su habitat con armonía, es así cómo esta arquitectura sin arquitectos demuestra que su "naturaleza áspera estimula la capacidad artística del hombre anónimo que la edificó" (Rudofsky 1968: 58). En su composición existen algunos elementos que Celia Barahona describe como "Ios revestimientos continuos constituyen la piel de los edificios y como tal su principal misión es la de proteger las fábricas resistentes" (La Spina 2012: 24), dichos revestimientos no son la solidez o soporte de la edificación, son elementos constructivos 
que se enfrentan a las condiciones atmosféricas y antrópicas del ambiente.

La elaboración de estos morteros de revestimiento ha llegado hasta nuestros días cual dogma de fe y se han asumido sin el más mínimo cuestionamiento; no nos hemos preguntado si funcionan bien, si poseen la dosificación necesaria, si pueden aplicarse como mortero de sustitución sobre superficies patrimoniales, entre otras; Ruiz de Santayana (1922: 284) mencionó con gran determinación "quienes no pueden recordar el pasado están condenados a repetirlo". Actualmente, estas intervenciones para salvaguardar el patrimonio se encuentran enmarcadas en la historia de los hechos en sus antecedentes, sus rasgos sociales, su cosmovisión, etc. Sin embargo, el pasado histórico y constructivo, su materialidad y las características particulares de sus componentes llegan a plasmarse en un informe mas no en una investigación a profundidad, no obstante, el documento es un punto de partida para análisis comparativos e información histórica de elementos que en algunos casos ya no existe. En el caso de Quito se han aplicado técnicas empíricas, análiticas y academicistas en la restauración de las edificaciones, se ha investigado poco y las publicaciones son escasas. Este no es un hecho aislado o una interpretación personal ya que Galeano (1998: 181) lo comentó en su escrito Mapamundi "La geografía tradicional roba el espacio, como la economía imperial roba la riqueza, la historia oficial roba la memoria y la cultura formal roba la palabra"; nace ahí una inquietud, ¿qué pasa con nuestra memoria constructiva? Las intervenciones modernas en edificaciones patrimoniales han roto la concepción de salvaguardar el bien inmueble en correspondencia con las técnicas utilizadas durante siglos, actualmente se recurre al uso combinado de materiales utilizados en el pasado o técnicas vernáculas, a las que se añaden materiales actuales que se comercializan en el mercado, sin un análisis previo, cuidadoso y respetuoso de bien patrimonial. En otros casos, el informe cumple con los requisitos para la aprobación del proyecto de intervención arquitectónica que es el principal interés; por otra parte, los contratos de estudios tienen duración corta y presupuestos bajos, lo cual genera un resultado incompleto que no contempla la consolidación íntegra del bien inmueble, ni de sus componentes, es decir, la caracterización de los mampuestos, revestimientos, etc. Sólo se repara y a veces se mal repara, es por esto que la preocupación sobre el mortero de revestimiento es imperceptible ya que a menudo se lo remedia con materiales incompatibles y de fácil solución, es una cadena de enseñanza mal engranada entre la teoría y la práctica. Aplicaría aquí lo que Eric Arthur Blair decía "para hacer cumplir las mentiras del presente, es necesario borar las verdades del pasado" (Orwell 1984: $29,141)$. El proyecto investigativo trata de delinear el perfil constructivo del mortero de revestimiento utilizado en las construcciones de Quito durante los siglos XVI, XVII y XVIII, apoyándose en la caracterización de los materiales. Se dará un detalle físico, mecánico, químico y mineralógico de los morteros originales extraídos del sitio, en este sentido, los estudios de caracterización de los materiales, "proporcionarán resultados que servirán para establecer la datación relativa de las muestras" (Rojo 2015: 42), y así poder generar una correlación entre la historia disciplinar y la historia constructiva de los casos de estudio, tomando en cuenta que esta arquitectura es parte de un proceso de colonización procedente del viejo mundo y de saberes constructivos locales.

Como base metodológica se ha tratado de reunir todos los insumos necesarios diferenciando aquellas fuentes primarias de las secundarias $u$ otras evidencias que han contribuido a la investigación, tratando de no alterar el objetivo de la investigación que es determinar el tipo de mortero de revestimiento que se utilizó en las construcciones del Quito colonial. La fuente primaria es el edificio mismo, ya que las muestras son tomadas en el sitio, previo un análisis de los lugares menos intervenidos, este material es sometido al análisis y composición de los morteros partiendo de la macro a la micro localización. Las fuentes secundarias son textos sobre la historia del edificio, información de periódicos y revistas; este material es confrontado con el análisis científico de materiales extraídos en el laboratorioy los resultados son confrontados con investigaciones similares sobre la caracterización de morteros, con la finalidad de argumentar mejor sobre los problemas planteados en un principio.

\section{El uso de los morteros en la historia}

Sobre los morteros históricos hay múltiples investigaciones, tanto desde el punto de vista descriptivo como el analítico. Como preámbulo, vale decir que esa combinación simple a base de tierra cruda mezclada con ceniza volcánica o coprolitos que se evidencian en la construcción de muros, suelos y pavimentos en el Neolítico son la base compositiva de un mortero, o aquella argamasa de arcilla que al añadir agua permite su manipulación adquiriendo gran dureza al secarse (Sersale 1991: 259); o por qué no enunciarlo, el uso de los morteros de cal que se remontan a un terrazo excavado en Canjenu, actual Turquía, que posee un mortero de cal fechado entre 12.000 a.C. y 5.000 a.C. Sin embargo, en el desarrollo de la historia antigua, tanto de Asia como de Europa es más habitual el uso de morteros de cal y yeso (Elsen 2006: 1417). El uso exclusivo sólo de mortero de cal se remonta a la Máscara de Jericó 7.000 a.C., actual Palestina, donde el empleo de enlucidos y empastes a base de cal les permitió experimentar con áreas pulidas y de gran acabado superficial (Malinowski 1981: 342), y si describimos con más detalle debemos enunciar la aldea Catl Hüyük 6.000 a.C., actual Turquía, donde se recubren muros con un pequeño empaste que va desde la tierra mezclada con elementos orgánicos e inorgánicos hasta conglomerantes de yeso y cal (Álvarez, Pérez y García 1995: 52).

Si nos referimos a la adaptación de materiales de la localización, los pueblos que se asentaron en la antigua Mesopotamia, humedecidos por los márgenes del Tigris 
y Éufrates, dada su úbicación geográfica optaron por una argamasa rica en arcillas y betumen enunciada ya en el Génesis "... y se dijeron unos a otros: Vamos, hagamos ladrillo y cozámoslo con fuego. Y les sirvió el ladrillo en lugar de piedra, y el asfalto en lugar de mezcla. Y dijeron:Vamos, edifiquémonos una ciudad y una torre..." (Varela 1869) que no sólo les sirvió para el uso de elementos edificados, sino para realizar construcciones de gran envergadura como el templo de Ishtarat, Ninni-Zaza, 1800 a.C. donde se resalta el uso cromático del betún y la cal e incluso el yeso; o la Torre de Babel 800 a.C. con la construcción de ladrillos y betún que se usó como mortero de junta, entre otros (Alejandre 2002: 12-13).

Se puede afirmar que en el antiguo Egipto fue donde se desarrolló el mayor conocimiento constructivo con yeso. Las piezas megalíticas de roca de las pirámides tuvieron un empaste de yeso que les permitía generar su decoración pictórica; este pueblo también lo utilizó como una lechada que servía como deslizante y lubricante de las grandes superficies rocosas en sus construcciones (Furlan y Bissegger 1975: 166), e incluso dicho conocimiento les permitió usar el yeso como ligante en los intersticios de sus juntas constructivas (Martinet, Deloye y Golvin 1992: 40).

Son los griegos los que viven una arquitectura evolucionada justificada en la filosofía, ciencia y técnica, y exponen los elementos arquitectónicos y constructivos con gran equilibrio y sensatez; siendo así que las primarias construcciones de piedra estaban unidas por un mortero hecho simplemente de tierra o de arcilla (Orlandos 1966: 135-148). Ya Vitrubio escribió el gran trabajo sobre los elementos constructivos por la selección de materiales, elaboración y técnica de aplicación. Con el transcurso del tiempo y la mejora de la técnica, en los griegos predominó el uso de los morteros de cal tanto como ligante como para acabado superficial. Esta técnica en la cumbre de su cultura constructiva logró generar morteros pulidos: establecieron acabados superficiales por la sucesión de tres a seis capas hasta lograr un estuco consolidado a base de cal de alta resistencia que aumentaba la impermeabilidad del material, la misma que se incorporaba visualmente con la superficie del mármol (Furlan y Bissegger 1975: 167; Malinowski y Garfinkel 1991: 62). Incluso las investigaciones manifiestan que los griegos conocían los ligantes artificiales, lo que corrobora la hipótesis del origen neolítico del mortero de cal. Alejandre describe en sus investigaciones que la arqueología clásica evidenció la utilización de morteros de cal al exterior "revocos" o al interior "enlucido" en restos de construcciones como Cadmos en Tebas, el Megaron de Pylos, el Palacio de Micenas así como el Palacio de Tirinto, que se encuentran datadas aproximadamente en el 1.400 a.C. con la caída de Cnosos (Alejandre 2002: 15). La experimentación fue tal que debido a la falta de rocas volcánicas en el sitio utilizaron puzolánico artificial extraido de los tejuelos, ladrillo triturado o la ceniza de la cáscara de arroz; esta experimentación de añadir nuevos componentes les permitió fortalecer y generar morteros de resistencia hidráulica (Válek, Hughes y Groot 2012: 130).

El Imperio Romano, en todos sus periodos de expansión, se nutrió del conocimiento constructivo de sus pueblos conquistados, "aunque la cal tuvo un profuso empleo con anterioridad a los romanos, podemos afirmar que fue la civilización romana la que mejoró los procesos de fabricación de la cal y las técnicas de la puesta en práctica de los morteros, y supo explotar todas las posibilidades de este material y además popularizó y expandió está técnica por todo el imperio" (Gárate 1993: 59). Refleja con mayor fuerza la herencia de la tecnología constructiva griega en el empleo de mortero de cal Opus Caementitium, dejando a un lado los primarios sistemas como el de gruesos bloques hilados sin morteros Opus Quadratum o los de ladrillo seco Opus Latericium y desarrollaron el uso frecuente del mortero multicapa en obras monumentales como el Panteón, Coliseo y sus acueductos (Malinowski y Garfinkel 1991: 62). Se llegaron a mejorar los morteros romanos perfeccionando la argamasa desde la selección de la piedra de cantera hasta la cocción, sin olvidarse del uso de diversas sustancias como escoria, carbón, etc.; lo que les facultó tener una mezcla con mejor consistencia, durabilidad, impermeabilidad denotando con esto que su mortero hidráulico posee mejores características que su antecesor griego (Válek, Hughes y Groot 2012: 133; Morgan 1992: 11-15). Mientras tanto, esto les permitió mejorar los procesos de fabricación de la cal y las técnicas de aplicación de su mortero, bien por el empleo de cal como por la adición de otro tipo de áridos: pétreos, cerámicos e incluso puzolánicos, como fue la tierra volcánica (Rassineux, Petit y Meunier 1989: 1027). El desarrollo de la técnica del mortero romano fue tal que permitió desarrollar nuevos parámetros de acabados constructivos para ser aplicados sobre las superficies de los muros; así se tiene un recubrimiento impermeable multicapas con espesor decreciente llamado Opus Signinum que se aplicaba sobre grandes rocas que se exponían a la intemperie como es el caso de las cloacas, o el estuco compuesto por cal hidráulica y polvo de mármol Opus Marmóreum el mismo que se empleaba sobre superficies irregulares para aplomar y emporar, sin olvidar aquel empaste fino de cal grasa con el cual se blanqueaban las paredes y se pintaban sobre las mismas los frescos o murales el Opus Abarium (Ortega 1994: 48-51).

En el intervalo de esta etapa de la historia están los morteros bizantinos, visigodos e islámicos: los morteros bizantinos, de entre el siglo III al XIII se caracterizaron por tener morteros de cal cuya mezcla era tosca y gruesa aplicados sobre la construcción de sus muros, vale añadir que en el proceso de aplicación de los morteros sobre la superficie se incorporaba polvo de ladrillo o cerámico antes del fraguado como una idea burda de mejorar la consolidación y durabilidad del material; mientras que la generalidad de los morteros visigodos era mantener la línea y técnica constructiva de la arquitectura romana tardía donde el ligamento de cal es imprescindible para 
las juntas y superficies de sus sillares (Alejandre 2002: 22-27). Los morteros islámicos diferencian bien el uso y el trabajo de cada uno de los elementos constructivos, mezclas de tierra, cal y yeso; es más, lo tenían registrado en su Ordenanza de oficios y mercados o reglamento del Zoco, siglo XIV, el hisba o la sharia que prohibía el uso de materiales inadecuados, baratos, e incluso, todo tipo de comercialización que ponga en tela de juicio la calidad del pétreo a utilizar (Michell y Grube 1985: 174185). Con respecto al uso de la cal se puede manifestar que desarrollaron tres usos comunes con este material, estos fueron el tapial monolítico de cal, el tapial real y el tapial calicastrado, en los que se determina el uso con respecto a la cantidad y espesor de capas de mortero y su dosificación, esta caracterización en la tapia es lo que determina su eficacia, sin olvidar la decisión del uso de la cal para impermeabilización de superficies como arranque de muros, drenajes, canales de agua y techumbres; en este período era habitual el uso de paja como aditivo para mejorar la resistencia de las argamasas de mortero (Ontiveros-Ortega, Sebastián-Pardo, y Valverde-Espinosa 1999: 49). Respecto al yeso se puede manifestar que en la época islámica este material fue el componente ideal para las argamasas de morteros de junta y revestimiento, el motivo principal, la abundancia del mismo en oriente medio del norte de África y el sur de la Península Ibérica así como la menor temperatura de cocción comparada con la cal (Alejandre 2002: 23); el mismo se prefería para estucos de gran precisión y acabado fino. Los primeros usos del yeso se registran como enlucido interior de las casas colmena del norte de Siria; la utilización del mismo representa un impulso interesante en la Península Ibérica islamizada donde la edificación religiosa se nutrió de esta aplicabilidad del material entre los siglos VIII al XV, con ejemplos de elegancia arquitectónica como la Mezquita de Córdoba bajo el Emirato de Abderramán I, el Palacio de la Aljafería de Zaragoza, Alcazaba de Balaguer en Mérida, o el esplendor constructivo de la Alhambra de Sevilla, básicamente toda aquella riqueza estética en yesería del periodo al-Andalus (Sanz 2009: 16-20).

En el medioevo, después de la caída del Imperio Romano, fue difícil conservar el acervo de conocimiento sobre los morteros; es más, cada región aplicó y varió la utilización de los principios romanos adaptándolos a su necesidad local, matizándolas o alterándolas con la experimentación de nuevas fibras, aditivos y agregados, lo que derivó en morteros de composición química y mecánica diferentes (Newton y Sharp 1987: 163-175). El medioevo español es una mezcla serena y estética donde predomina la construcción clerical, los castillos y fortificaciones militares, se evidencian morteros a base de cal con sillares de piedra en las juntas, se preparaba un mortero con lajas de piedra o trozos de teja para evitar la introducción de troncos de madera y hacer palanca. En Italia, Francia, España así como en el resto de países que sostuvieron el pensamiento del medioevo, existió un retroceso en los procedimientos y la calidad en la fabricación de la cal, pues declinaron en la selección granulométrica del árido, la temperatura de cocción, la proporción y homogeneidad de las mezclas, desvinculando la relación entre el ligante y el agregado (Gutierrez-Solano et al. 1989: 45; Malinowski 1981: 342; Furlan y Bissegger 1975: 169). Esta deficiente conceptualización del mortero intentó mejorarse con aditivos orgánicos como albúmina de huevo, caseína de mamíferos, queratina de animales, cera de abejas, gluten de trigo - centeno - cebada, zumo de frutas, fibra o aceite vegetal, sangre animal y urea - orina (Sickers 1981: 25-52; Rampazzi et al. 2015: 115-116). No obstante, en el mismo estudio se concluye que el uso de estos aditivos conllevan a la aparición de microorganismos que originan patologías químico biológicas en los elementos constructivos. Sobre el ocaso del medioevo, por decreto monárquico o aristocrático muchos países estimulan la construcción en piedra, la misma que siglos antes estaba reservada a edificios religiosos y militares, con la finalidad de que perduren en el tiempo y eviten ser la antorcha de la hoguera en la conquista de sus pueblos; obviamente esto cambió el hábito de los constructores de módulos pequeños y unifamiliares pues tuvieron que adaptarse a las exigencias del sistema constructivo ignorando las variaciones crecientes de calidad del pétreo y de la cal, decreciendo la eficacia de la fabricación del mortero (Furlan y Bissegger 1975: 169).

Posterior a la descripción que se ha realizado, los empastes, morteros y revestimientos han ido manteniendo el mismo principio de composición con la variable lógica de adaptación regional a su geo localización y estilo arquitectónico con variaciones plásticas del elemento constructivo a larga duración. Se ha pasado de los morteros prehistóricos rústicos y toscos hasta aquellos revocos y enlucidos decorativos en el auge de la burguesía - la experimentación de ligantes hidráulicos con el empleo de la cal resistente a la acción del agua marina, los cuales generaron un nuevo esquema de utilización del material constructivo, algo que ya realizaron los griegos y romanos siglos atrás (Álvarez, Pérez y García 1995: 58). No serán parte del estudio los morteros actuales generados artificialmente o cementos como el portland, puzolánico, aluminoso, etc.

\section{El uso de los morteros en américa}

Es necesario poner sobre contexto que con el descubrimiento de América en 1492 se produjo la aplicación de materialidades ya experimentadas, donde existió una tradición contructiva prehispánica que tuvo su arraigo local y experiencia en la construcción y en el uso de materiales naturales en bruto. Todo esto se evidencia en el uso ornamental de los morteros con pigmentos y texturas, como el azul maya o el rojo de la cochinilla, producto de sus cactos centroamericanos, entre otros (Maganoli et al. 1995: 483-485). [figura 1] La llegada de los conquistadores y su conocimiento en técnicas y tratados constructivos generó un producto con variables etnotipológicas, el uso de materiales locales orgánicos e inorgánicos y la riqueza 


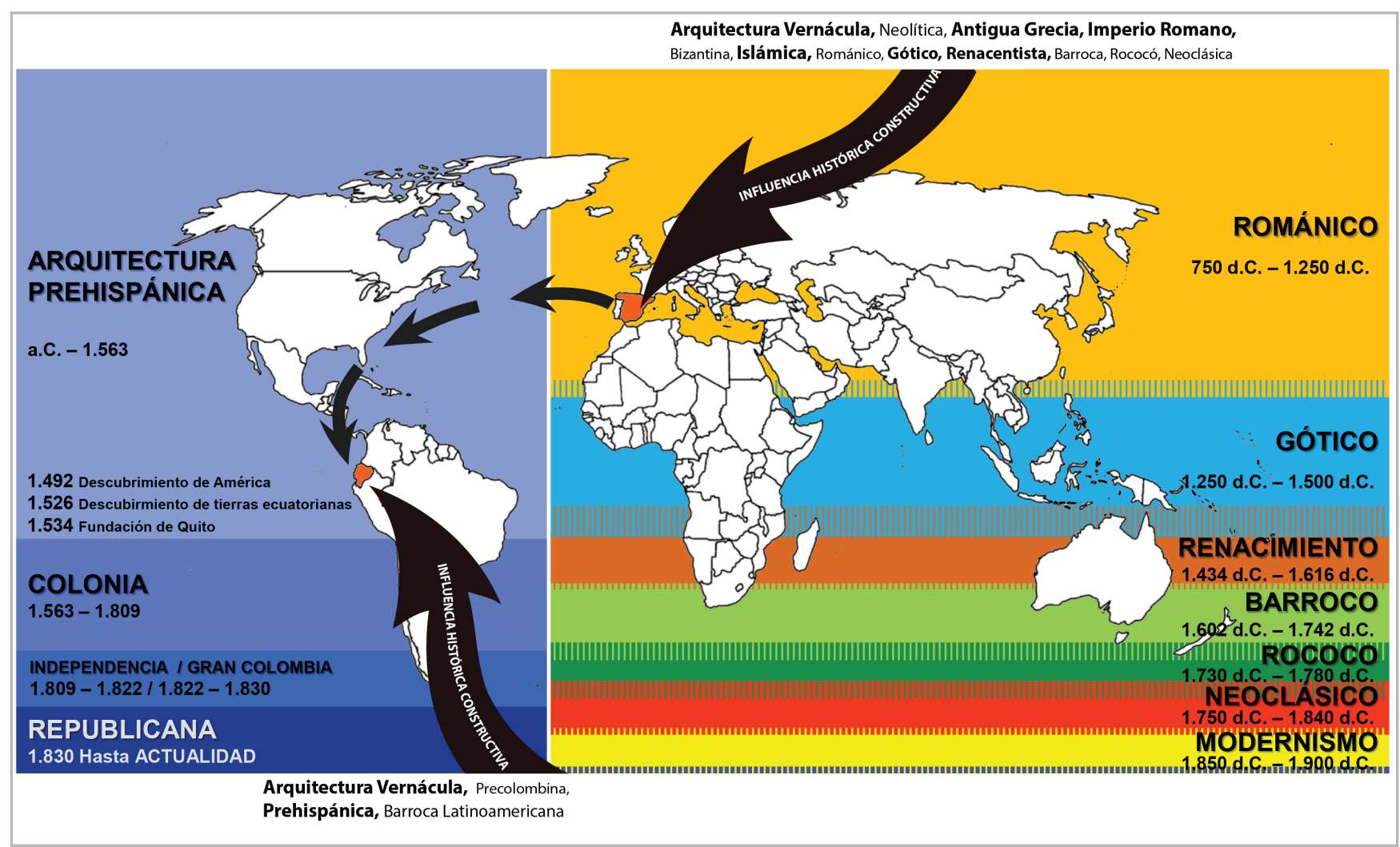

Figura 1.- Cronología de la influencia de los estilos de arte - arquitectura determinantes para poder delimitar los principios de una arquitectura ecuatoriana

experimental local se nutrió con los aspectos técnicos depurados de los morteros de cal (Martínez et al. 2008: 95). Al parecer, en el Imperio Inca el trabajo con argamasas, morteros de junta o revestimientos fueron poco utilizados, en tal caso un mortero de barro sería una de las escasas alternativas (Ramón 2017:300-301), sin embargo, ciudades como Chan Chán en la costa norte del Perú y Pachacámac en Lima, son ejemplos significativos de uno de tierra. En la configuración de su arquitectura monumental inca la piedra manifiesta el poder del pueblo, la utilización simple de una mezcla rústica a base de tierra cruda como lo evidencian sus vestigios arqueológicos "con restos de coprolitos, se define un estrato compuesto por grava, arena gruesa y tierra fina arcillosa" (Chu 2017: 30). En el florecer del imperio las investigaciones evidencian que las construcciones en el valle del Cuzco usaron morteros de barro y paja ichu (McEwan, Gibaja y Chatfield 2005: 259). Sin olvidar que agregados pétreos finos eran usados para utensilios domésticos. En América, la construcción a la española no se evidenciará sino con la práctica urbanística de la ciudad en Santo Domingo, República Dominicana, en 1494 (Ortiz 2004: 21).

\section{El uso de los morteros en ecuador y en la villa de Quito}

La llegada de los europeos a las costas del territorio ecuatoriano ha sido determinado en 1526, en ese momento la arquitectura vernácula refleja una respuesta arquitectónica afín con la organización social, los señoríos étnicos: trabajo colectivo, aporte estético y constructivo acumulativo que se refleja en el material constructivo básico que tiene cada región geográficay las características metereológicas zonales. Es así que la arquitectura monumental que expresaba el poder dominante se diferencia de la vivienda común por su presencia, materialidad y su larga duración. Los resultados de los estudios arqueológicos han permitido determinar como material predominante el uso de los morteros de tierra. No obstante, hace falta un estudio en profundidad de los 6500 años de construcción prehispánica. Los periodos prehispánicos, determinados ya en investigaciones en los años sesenta, son tres: el Periodo Formativo (3500 a.C. - 500 a.C.) que se caracteriza por el uso del sistema constructivo entramado de madera denominado bahareque, con postes de madera empotrados en el suelo practicados tanto en edificaciones de la costa como de la sierra; el Periodo de Desarrollo Regional (500 a.C. - 500 d.C.), caracterizado por el uso de cimientos y sobre cimientos de piedra con muros de bahareque en la costa y construcciones de madera y paja de páramo en la sierra; el Periodo de Integración (500 d.C. - 1534 d.C.) en el que se consolida la praxis de la construcción con cangahua, bahareque y piedra; construcción de pirámides truncadas y escalonadas de cangahua; pisos conformados por planchas de arcilla cocida en la cima de las pirámides, mediante la quema de material vegetal. Sobre la plataforma de arcilla cocida se encontraba una construcción de bahareque. En la costa la construcción en caña y madera se la realizó sobre montículos de tierra 
en las zonas húmedas para impedir que suban animales rastreros o depredadores (Del Pino 2018: 92).

La huella prehispánica constituye una preexistencia con la que cuentan los fundadores de ciudades del actual Ecuador, es así que Fray Gaspar de Torres para 1597 describe las construcciones de los Caranqui "las casas de los naturales son de un sistema de cañas, sin enlucido de barro y cubiertas con hojas de palma" (MDMQ 2003: 22); mientras Salinas de Loyola describe las costumbres constructivas del bohío de los Paltas en la zona de Loja "las casas donde habitan son de paredes de palos, o tablas y algunas cubiertas con barro a manera de tabique y otras de paja, la cubierta, en general de la misma paja..." (22). La Fundación de la villa de San Francisco de Quito se impone mediante una sucesión de guerras, saqueos, y finalmente la negociación entre españoles e indígenas al finalizar el año 1534 (Del Pino 2017: 165-169; Ortiz 2004: 23-29). La arquitectura residencial del Quito colonial se caracterizó por soluciones de una sola planta monoambiente como las prehispánicas, construida por su propio habitante, con el apoyo de la comunidad indígena. La construcción de este bohío usó "materiales rústicos sin procesamiento evidenciando el uso de barro, adobe, bahareque, madera o caña con cubiertas de palmas o paja sobre estructuras de madera rolliza, según el clima y la disponibilidad de los materiales" (Ortiz, Abram y Najera 2007: 102). En otras palabras, aseveramos la frase pronunciada en 1990 de Valerie Fraser sobre la arquitectura colonial andina "la arquitectura de la América española del siglo XVI es una arquitectura de y para la conquista; es fundamental para la construcción y consolidación del imperio español" (Webster 2011: 305).

El Centro Histórico de Quito se desarrolla sobre una topografía agreste y particular donde "las irregularidades del terreno y los errores en las medidas, dan por resultado pequeñas variaciones que conducen a un pequeño equilibrio entre la unidad y la diversidad" (Rudofsky 1968: 55). Emplazado en una área de 376 hectáreas, posee un total de 4674 predios edificados de los cuales 4286 se encuentran inventariados y de éstos 130 bienes son monumentales, entre los cuales figuran iglesias, conventos, claustros, etc., que representa el $42 \%$ de los predios edificados, mientras que el $58 \%$ restante equivale a construcción de arquitectura civil. La parroquia González Suárez representa el núcleo central de consolidación histórica, posee 54 hectáreas, es aquí donde se emplazan las edificaciones a estudiar (Del Pino 2010: 15).

Conocido como el Gran Convento, el Centro Histórico de Quito entre la época de su fundación en 1534 y mediados del siglo XVIII, tuvo alrededor de una trentena de edificaciones destinadas al clero mendicante estableciendo un vínculo entre el poder dominante y la producción arquitectónica. Alfonso Ortiz detalla que para el siglo XVI, con una población de 4.000 personas, 15 edificaciones religiosas sobresalían en este centro urbano colonizado (Carrión et al. 1990: 142). A pesar de la diversidad de órdenes religiosas y ejemplos de gran valor arquitectónico "la definición va a ser mayoritariamente monumentalista, y limitada geográficamente a algunas zonas: el centro histórico y unos pocos barrios más identificados por su riqueza arquitectónica" (Burgos-Vigna 2015: 67).

En las investigaciones publicadas sobre la arquitectura y la ciudad de Quito describen la utilización progresiva de los morteros. En un principio, cuando la construcción era de mampuestos de tierra y piedra, se utilizaron morteros de tierra cangahua (Boada et al. 1993: 83). Esta no es sólo una aproximación local pues investigadores han evidenciado este material como una respuesta doméstica primaria "el barro parece ser el primer material de unión empleado para fabricar morteros en un edificio antiguo, que está en uso, hasta ahora en algunas zonas del mundo" (Moropoulou, Bakolas y Anagnostopoulou 2005: 295). Luego, con el pasar de la conquista y el asiento del dominio español se optó por "blanquear con cal las paredes interiores y exteriores de las casas" (Boada et al. 1993: 111). Esta práctica se explicará más tarde por la necesidad de asepsia en las ciudades y haciendas afectadas por plagas y pestes.

En los estudios que Susan Webster hace sobre la Iglesia San Francisco comenta que "se observa una mezcla de piedras de cantera de cal y arena" (Webster 2012: 50) [figura 2]. Este es un primer indicio que pone en alerta la

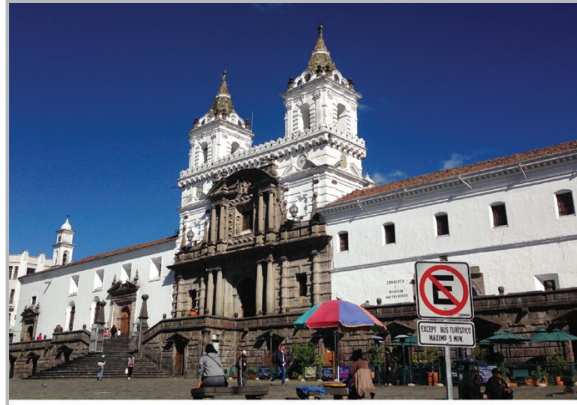

Mortero de cal sobre fachada Iglesia San Francisco

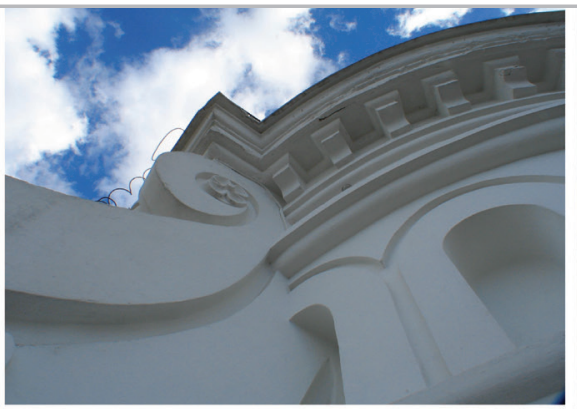

Mortero de cal en cúpula de Catedral Quito

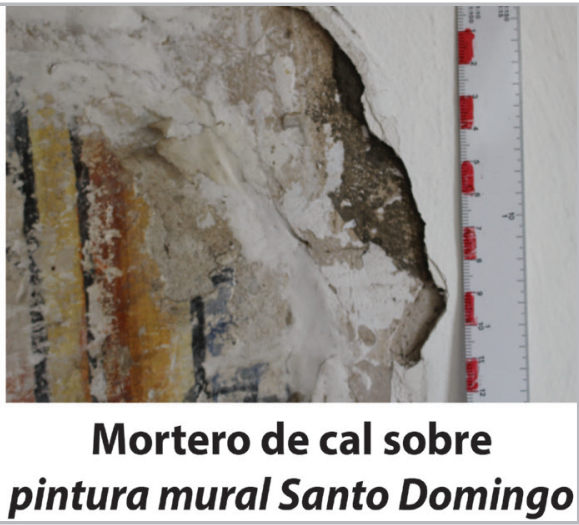

Figura 2.- Ejemplos de revestimientos encalados 

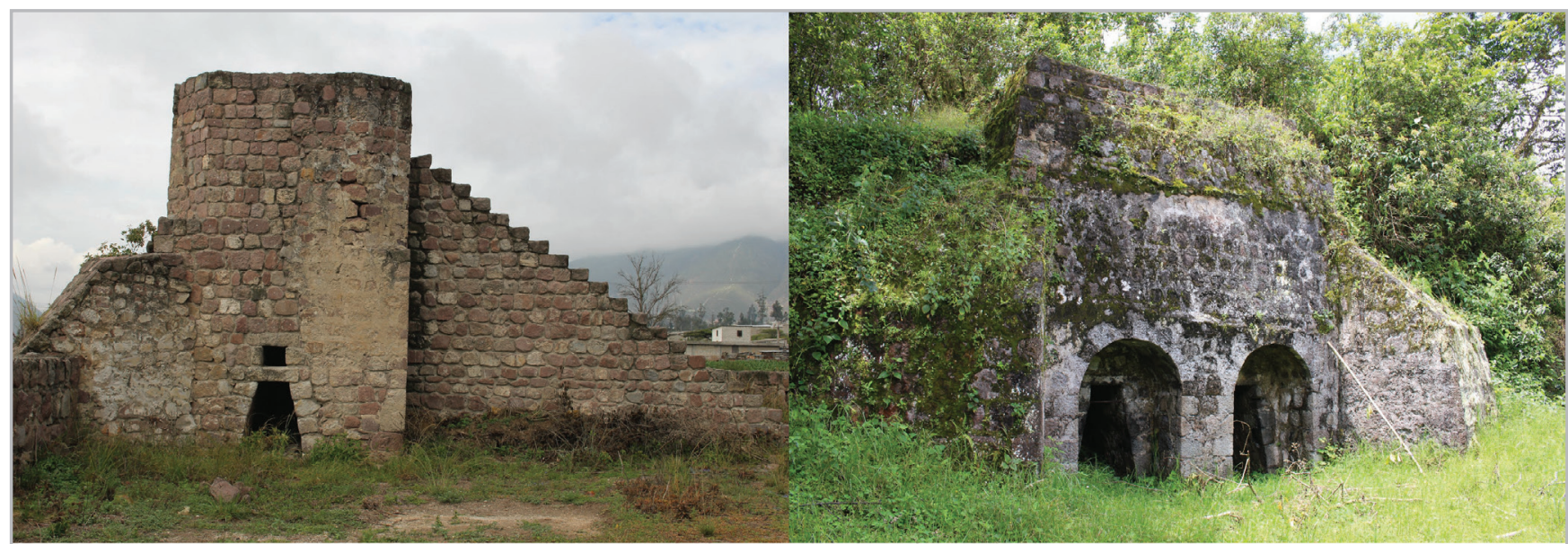

Horno de Cal "Rumicucho"

Horno de Cal "Reserva Geobotánica Pululahua"

Figura 3.- Hornos antiguos de Cal

existencia de morteros a base de cal para unir, consolidar y dar un acabado que determina una estética a los muros de mampostería; no obstante, dentro del proceso constructivo, desde la colonia hasta la actualidad, no existe una tabla de elementos ni de dosificaciones, sino únicamente la aplicación empírica "prueba error de dosificaciones de la mezcla" esperando la compatibilidad del mortero nuevo sobre la superficie ya construida. En el estudio del Proyecto de Cooperación Internacional EcuadorBélgica, Ecua-Bel, se menciona que en la Iglesia Santo Domingo se desarrollaron prospecciones arqueológicas que determinaron un abanico de información no muy ordenada que identifican los sistemas constructivos y los materiales empleados, pero no establecen una cronología histórica constructiva; por ejemplo en el informe que transcribe Kennedy dice "este noviciado... elegante y espacioso, fue construido todo en cal y ladrillo sobre un terraplén en arquería de cal y piedra" (Alemán y Van Balen 1994: 36), pero los informes de restauración mencionan el uso de morteros puzolánicos en proporciones elevadas. Una de las explicaciones de esta asimetría en la información se encuentra en la reforma dominicana aplicada a las iglesias de la Orden en toda América; los daños producidos por sismos y terremotos posteriores a la construcción del edificio, permiten identificar que la mayor parte de los edificios religiosos monumentales del centro histórico de Quito tienen hasta tres reconstrucciones y en algunos casos profundas reformas en las que los sistemas constructivos son los mismos utilizados durante siglos. El cambio se produce con la inserción del cemento puzolánico cuya difusión es notoria desde mediados del siglo $\mathrm{XX}$, aspecto que debe ser estudiado en profundidad para determinar con mayor precisión las etapas de la arquitectura monumental religiosa de Quito.

La construcción civil se identificó por la edificación en tierra con sistemas como el adobe, tapial y bahareque considerando que al menos el adobe tuvo una semejanza métrica al adobón de Andalucía "fabricado en el propio terreno... de largas dimensiones, más o menos de 1.20m de largo por 0.70m de alto" (Navarro 1985: 36,135). Para proteger la construcción de tierra se la recubrió con una lechada compuesta por tierra muy fina y agua para rellenar las fisuras producidas por el secado del muro; el material térreo para la construcción se lo denomina en Ecuador chocoto. Posteriormente, "la necesidad de enlucir y revestir las paredes con cal para preservarlas de las inclemencias del tiempo" (37). Para 1541 los fundadores tuvieron ya algunos materiales de construcción para mejorar las edificaciones originarias, en 1537 se comenzó a "explotar cal en un pequeño horno de Tolóntag" (Enríquez 1938: 217), pero el Quito construido con cal y canto se consolidó hacia 1551, año en que Francisco Ruiz obtiene los permisos para hacer y utilizar las minas de cal en Tolóntag y posteriormente a esta mina se suma la de Lulumbamba y Calacalí (Enríquez 1938: 235; Andrade-Marín 2003: 119-120). [figura 3] Desde ese momento la cal permitió generar esa argamasa que consolidaría la construcción de obra monumental o civil en la ciudad así como la infraestructura: pavimentos, puentes, arquería y algunas cloacas subterráneas de las quebradas que fraccionaban para ese momento este centro urbano.

El conocimiento progresivo del uso de la cal se impuso entre los siglos XVI y XVIII, adaptándose a la necesidad que imponía el orden colonial, a las condiciones de clima y humedad, al imaginario histórico constructivo y colectivo que asoció la mampostería de piedra, tierra y ladrillo cocido encalados como un rasgo de prestigio en la arquitectura y un patrón racional de construcción. [figura 4] El propósito de la investigación doctoral titulada Caracterización de los morteros de revestimiento del Quito Colonial en los siglos XVI, XVII y XVIII es identificar los tipos de morteros usados entre estos siglos, reconocer su composición, calidad, estética y modo de preparación como parte de la valoración de las edificaciones históricas patrimoniales. En los siglos XIX y XX la utilización del mortero de cal es parte de la cotidianidad en la prática constructiva de la arquitectura republicana (Boada et al. 1993: 136). Pero, este material 


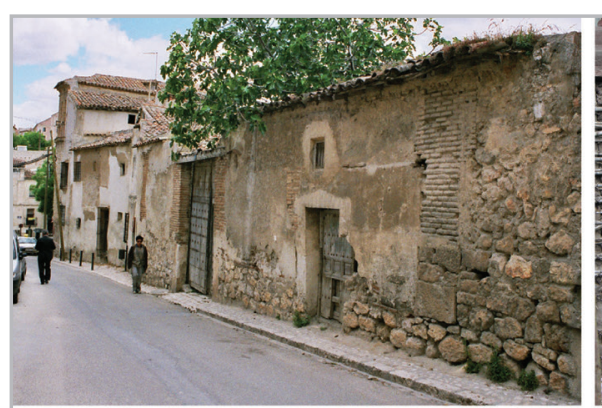

Mortero de barro sobre muro piedra y ladrillo

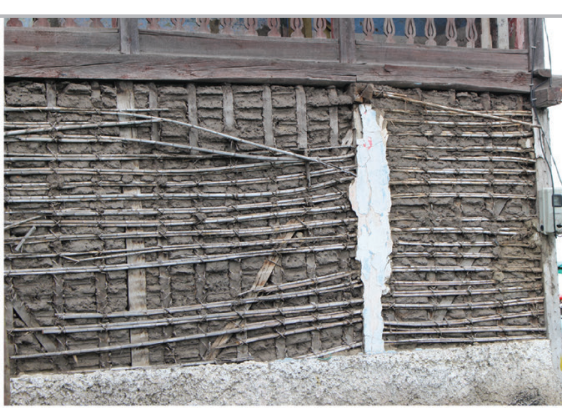

Mortero de barro y cal sobre muro bahareque

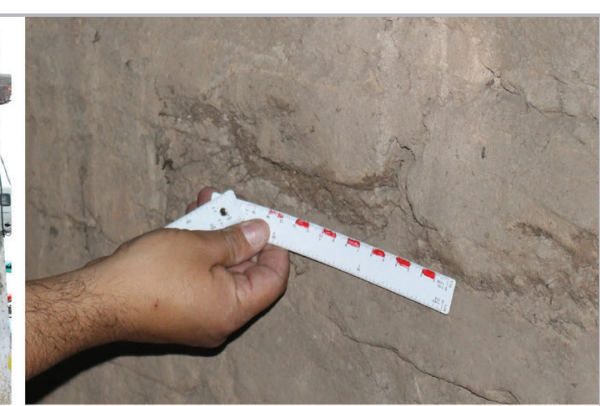

Mortero de barro sobre muro adobe

Figura 4.- Revestimientos de barro

¿fue usado por las ventajas de impermeabilidad, plasticidad y biodegradación o como un principio simplemente estético o fachadismo instaurado por el imaginario colectivo u ordenanzas locales?. Hay que tener en cuenta que los morteros de revestimiento por su empleo son la "piel a sacrificar" (Baldi et al. 1981: 134), pues están en constante contacto directo con agentes que alteran su composición.

\section{Conclusiones}

La investigacion demuestra una primera aproximación de la utilización de morteros tradicionales de barro, otros de arena e hidrato de cal y de morteros de cal y yeso (Castilla 2004: 13), aplicados sobre los mampuestos. Esta reseña de hechos descriptivos y empíricos nos permitirá en posteriores etapas considerar fases deductivas, analíticas e investigativas a partir de la información y los resultados obtenidos. "Una buena intervención comienza cuando se reúnen todos los argumentos" técnicos y constructivos "desde la ubicación, hasta las particularidades históricas constructivas propias de la edificación, sin olvidar el establecimiento de un programa de mantenimiento periódico del inmueble edificado" (Lara 2017: 39). Hay que tener en cuenta que en la investigación histórica sobre los constructores de los edificios religiosos de Quito existe un sesgo con respecto a la manera de describir la historia constructiva, omitiendo el proceso o elaboración de los morteros y negando la participación de artesanos locales en la edificación colonial bajo la denominación de "autores anónimos" cuando en realidad, en los libros de cuentas aparecen contratos, a veces con montos significativos, para la construcción de obras de cantería, carpintería, albañilería, entre otros oficios, como lo describe la investigación de Susan Webster.

En 1981 el Centro Internacional de Estudios para la Conservación y la Restauración de los Bienes Culturales (ICCROM), trató de estandarizar un mortero de restauración bajo ciertas características físicas, mecánicas, térmicas, de fraguado, de trabajabilidad, de porosidad, etc. (Prado et al. 2009: 2). Sin embargo, pese a este intento de normalización se tiene que considerar lo que manifestó Alessandrini en 1989 "no se puede uniformizar la metodología" ya que cada caso tiene sus propias particularidades basadas en el estudio histórico constructivo de la edificación. La materialidad, adherencia, composición, compatibilidad y la calidad del mortero de revestimiento, objeto de estudio, se someterán a un tipo de exploración sin olvidarse de la calidad del mantenimiento de la edificación (IAPH 2003: 43).

La arquitectura del centro histórico de Quito fue edificada por indígenas, mestizos, negros y alarifes europeos, quienes aplicaron y fusionaron las técnicas europeas con las locales. Esta práctica se aplicó según el tipo de construcción, la adaptación de técnicas a los materiales disponibles, a manera de ejemplo, en donde no hubo hierro, o este era muy costoso, se trabajó en madera, tanto las piezas como los ensambles; es decir, se dieron soluciones creativas para resolver problemas específicos del lugar mediante los recursos y las técnicas disponibles, visualizaron la lógica tras el poder de la conquista. En suma, el artesano local logró generar esa belleza yuxtapuesta, que hoy constituye la arquitectura patrimonial, como diría Jamshid Kooros “EI albañil en sus limitaciones encuentra posibilidades infinitas, creando con variedad y armonía; mientras que el arquitecto moderno, con todos los materiales y sistemas estructurales de que dispone, produce monotonía y disonancia"(Rudofsky 1968: 151).

\section{Agradecimientos}

Este trabajo ha sido posible gracias a la investigación del programa doctoral DTCA en Construcción y Tecnología Arquitectónica de la Universidad Politécnica de Madrid cuyo tema es: "Caracterización de los morteros de revestimiento del Quito Colonial en los siglos XVI, XVII y $X V I I I "$ el mismo que en el proceso investigativo determinó precisar un análisis cronológico de su predecesor constructivo en los morteros de revestimiento que se recopilan en este artículo. Partiendo de la premisa que el actual Ecuador fue parte del Virreinato del Perú, este dominio territorial, político y administrativo permitió aplicar y complementar algunas de las técnicas constructivas locales de manera empírica.

Agradecemosaaquellos compañerosquegenerosamente han compartido sus conocimientos sobre la historia de 
la arquitectura ecuatoriana, así como a investigadores de otras disciplinas, maestros de oficios que laboran en nuestras construcciones históricas y han aportado su conocimiento del uso, dosificación y composición de los morteros y revestimientos llamados coloniales; y más aún a los guardianes, priores y representantes técnicos de los claustros e iglesias que forman parte de esta investigación por la facilidad de acceso a sus instalaciones.

\section{Referencias}

ALEJANDRE SÁNCHEZ, F. J. (2002). Historia, Caracterización y Restauración de Morteros. Sevilla: Universidad de Sevilla, Secretariado de Publicaciones Instituto Universitario de Ciencias de la Construcción.

ALEMÁN, M., KOEN VAN BALEN, EDS. (1994). “Puesta en valor del Patrimonio Arquitectónico en el Convento de Santo Domingo". Vol. 5 de Estudios y Metodologías de Preservación del Patrimonio Cultural del PROYECTO ECUA-BEL (Ecuador - Bélgica). Bogota: Libri-Mundi.

ÁlVAREZ, J. I. MARTíN PÉREZ, A. GARCíA CASADO, P. (1995). "Historia de los morteros." Boletín informativo del Instituto Andaluz de Patrimonio Histórico 13: 52-59. https://doi. org/10.33349/1995.13.263

ANDRADE MARÍN, L. (2003). La lagartija que abrió la calle Mejía: Historietas de Quito. Quito: FONSAL (Fondo de Salvamento).

BALDI, P. CORDARO, M. MORA, L. MORA, P. (1981). "Architecture - "Couleur." Simposio ICCROM - International Centre for the Study of the Preservation and Restoration of Cultural Property, Roma, 3- 6 Noviembre, 133-144.

BOADA CASTRO, R., DEL PINO, I., GONZÁLEZ, M., LÓPEZ LÓPEZ, L., LUZURIAGA, M., MALDONADO, C., GABRIELA MERINO, et al (1993). "Arquitectura de Quito: Una visión histórica de su Arquitectura". Vol. 8 de Serie Quito. Quito: TRAMA, Dirección de Planificación del Ilustre Municipio de Quito, Consejería de Obras Públicas y Transporte, Junta de Andalucía.

BURGOS-VIGNA， D. (2015). "Del patrimonio a la cultura: evoluciones en la gobernanza urbana de Quito." Territorios 32 (32): 61-79. https://doi.org/10.12804/territ32.2015.03

CALDERON, L., LENIN, M. (2017). “Patología de la construcción en tierra cruda en el area andina ecuatoriana." AUC Revista de Arquitectura - Universidad Catolica Santiago de Guayaquil 38: 31-41

CARRIÓN, F., COLÓN CIFUENTES, I. P. FRANCISCO E., KINGMAN, E., NARANJO LALAMA, F., NARVÁEZ, A., et al . (1990). “Centro Histórico de Quito: Problemática y Perspectivas". Vol 1 de Serie Quito. Quito: TRAMA, Dirección de Planificación del Ilustre Municipio de Quito, Consejería de Obras Públicas y Transportes, Junta de Andalucía.
CASTILLA PASCUAL, F. J., MALDONADO RAMOS, L. (2004). "Estabilización de morteros de barro para la protección de muros de tierra" Tesis Doctoral en Arquitectura, Universidad Politécnica de Madrid.

CHU, A. (2017). "Investigaciones en el ushnu mayor de Incahuasi, Cañete: contribuciones al estudio de los ushnus incas." Revista del Instituto Riva-Agüero 2 (1) 11-56. https://doi. org/10.1007/978-0-387-76910-3 10.

DEL PINO MARTÍNEZ, I. A. (2010).“Centro Histórico de Quito: Una centralidad urbana en transformación hacia el turismo". Tesis de Maestría en Gobierno de la ciudad, Facultad Latinoamericana de Ciencias Sociales, FLACSO Quito.

DEL PINO MARTÍNEZ, I. A. (2017). “Espacio urbano en la historia de Quito: Territorio, traza y espacios ciudadanos". Tesis Doctoral en Arte y Arquitectura, Universidad Nacional de Colombia.

DEL PINO, I. (2018). “La huella prehispánica”, en M.D.M.Q., Quito Patrimonios (págs. 79-131). Quito: Azuca Cía. Ltda.

ELSEN, J. (2006). "Microscopy of historic mortars-a review." Cement and Concrete Research 36 (8): 1416-1424. https://doi. org/10.1016/j.cemconres.2005.12.006.

ENRÍQUEZ, E. (1938). Quito a traves de los siglos- Recopilación y notas bío-bibliográficas. Quito: Imprenta Municipal.

FURLAN, V., BISSEGGER, P. (1975). "Les mortiers anciens. Histoire et essais d'analyse scientifique." Zeitschrift für schweizerische Archäologie und Kunstgeschichte 32: 166. https://doi. org/10.5169/seals- 166356

GALEANO, E. (1998). Patas arriba: La escuela del mundo al revés. Madrid: Madrid Siglo XXI.

GÁRATE ROJAS, I. (2002). Artes de la cal. Madrid: Madrid MunillaLería.

GUTIERREZ-SOLANA, F., JAUREGUI, M., BOHIGAS, R., SARABIA, P. (1989). "Análisis de morteros medievales de dos castillos de Cantabria. España." Materiales de Construcción 39 (213): 37-45. https://doi.org/10.3989/mc.1989.v39.i213.815.

IAPH (Instituto Andaluz del Patrimonio Histórico) (2003). "Metodología de diagnóstico y evaluación de tratamientos para la conservación de los edificios históricos". Granada: Junta de Andalucía.

LA SPINA, VINCENZINA. (2012). “La metodología para el estudio de los revestimientos continuos tradicionales de las fachadas de los centros históricos." V Jornadas de Introducción a la Investigación de la UPCT, Cartagena, España, Abril: 24-26.

MAGALONI, D., R. PANCELLA, Y. FRUH, J. CANETAS, AND V. CASTANO. (1995). "Studies on the Mayan Mortars Technique." Material Research Society Symposium Proceedings. 352: 483489. doi:10.1557/PROC-352-483. 
MALINOWSKI, R. (1981). "Ancient mortars and concretes Durability aspects." Artículo presentado en el Simposio ICCROM - International Centre for the Study of the Preservation and Restoration of Cultural Property, Roma, 3 - 6 Noviembre, 341350.

MALINOWSKI, R., GARFINKEL, Y. (1991). "Prehistory of Concrete." Concrete International - American Concrete Institute (ACI) 13 (3): 62-68.

MARTINET, G., DELOYE, F.X., GOLVIN, J.C. (1992). “Caractérisation des mortiers pharaoniques du temple d'Amon à Karnak." Bulletin de liaison des laboratoires des ponts et chaussées 181: 39-45.

MARTINEZ, W., ALONSO, E. M., RUBIO, J. C., BEDOLLA, J. A., VELASCO, F. A., TORRES, A. A. (2008). "Comportamiento mecánico de morteros de cal apagada artesanalmente, adicionados con mucílago de cactácea y ceniza volcánica, para su uso en restauración y conservación de monumentos coloniales." Revista de la construccion 7 (2): 93-101.

MCEWAN, G., GIBAJA, A., CHATFIELD, M. (2005). "Arquitectura monumental en el cuzco del periodo intermedio tardío: evidencias de continuidades en la reciprocidad ritual y el manejo administrativo entre los horizontes medio y tardío." Boletín de Arquitectura PUCP (9) 257-280.

MICHELL, G., GRUBE, G. (1985). La arquitectura del mundo islámico: su historia y significado social. Madrid: Madrid Alianza Editorial.

MORGAN, G. C. (1992). "Romano - british mortars and plasters." Tesis doctoral en Arqueología e Historia Antigua, University of Leicester.

MOROPOULOU, A., BAKOLAS, A., ANAGNOSTOPOULOU, S. (2005). "Composite materials in ancient structures." Cement and concrete composites 27 (2): 295-300. https://doi.org/10.1016/j. cemconcomp.2004.02.018.

MUNICIPIO DEL DISTRITO METROPOLITANO DE QUITO, EMPRESA DEL CENTRO HISTÓRICO DE QUITO, Y FUNDACIÓN CASPICARA. (2003). Casa Ecuatoriana. Quito: Cobol Servicios Gráficos.

NAVARRO, J.G. (1985). Artes plásticas ecuatorianas. Quito: Banco Central del Ecuador.

NEWTON, R. G., SHARP, J. H. (1987). "An investigation of the chemical constituents of some renaissance plasters." Studies in conservation 32 (4): 163-175. https://doi.org/10.1179/ sic.1987.32.4.163.

ONTIVEROS-ORTEGA, E., SEBASTIAN-PARDO, E., VALVERDEESPINOSA, I. (1999). "Deterioration in XI-XIV century arab ramparts (Granada, Spain)." Materials and structures/Materiaux et constructions 32 (215): 45-51. https://doi.org/ 10.1007/ bf02480411.
ORLANDOS, A. K. (1966). Les matériaux de construction et la technique architecturale des anciens grecs. Paris: Editions E. de Boccard.

ORTEGA ANDRADE, F. (1994). "La Construcción Romana (1)". Revista de Edificación RE (18): 45-59.

ORTIZ CRESPO, A. (2004). “Origen, traza, acomodo y crecimiento de la ciudad de Quito". Quito: Fondo de Salvamento del Patrimonio Cultural de Quito (FONSAL), TRAMA.

ORTIZ CRESPO, A., ABRAM, M., SEGOVIA NAJERA J. (2007). "Damero". Quito: Fondo de Salvamento del Patrimonio Cultural de Quito (FONSAL), TRAMA.

ORWELL, G. (1983). 1984. Barcelona: Barcelona Círculo de Lectores ALMA.

PRADO GOVEA, R.H., CERECEDA, M.L., SPAIRANI-BERRIO, Y., HUESCA TORTOSA, J. A. (2009). "Estudio y caracterización de morteros para la restauración arquitectónica". CONPAT - Congreso Latinoamericano de Patología de la Construcción, Valparaíso, 29 Septiembre - 02 Octubre. http://hdl.handle. net/10145/12207.

RAMÓN, G. (2017). "Shaping precolonial concepts in the andes: The ushnu for Llocllayhuancupa (Huarochirí, Lima)." Latin american antiquity 28 (2): 288-307. doi:10.1017/laq.2017.22.

RAMPAZZI, L., COLOMBINI, M.P., CONTI, C., CORTI, C., LLUVERAS-TENORIO, A., SANSONETTI, A., ZANABONI, M. (2015). "Technology of medieval mortars: An investigation into the use of organic additives." Archaeometry 58: 115-130. https://doi. org/10.1111/arcm.12155.

RASSINEUX, F., PETIT, J.C., MEUNIER, A. (1989). "Ancient analogues of modern cement: calcium hydrosilicates in mortars and concretes from gallo-roman thermal baths of western France." Journal of the American Ceramic Society 72(6): 10261032. https://doi.org/10.1111/j.1151-2916.1989.td06263.x

ROJO ALVAREZ, A. (2015). "El análisis de morteros históricos como herramienta de datación e interpretación de técnicas y fases constructivas." Tesis Doctoral en Geología, Universidad de Oviedo.

RUDOFSKY, B. (1968). Arquitectura sin Arquitectos. Madrid: Editorial Universitaria de Buenos Aires.

SANTAYANA, G. (1922). The Life of Reason. Nueva York: Charles Scribner's Sons.

SANZ ARAUZ, D. (2009). "Análisis del yeso empleado en revestimientos exteriores mediante técnicas geológicas" Tesis Doctoral en Geología, Universidad Politécnica de Madrid.

SERSALE, R. (1991). "Lime, the first in time among the justly named binding materials." Atti della Accademia Pontaniana 40: 257-275. 
SICKERS, LAUREN-BROOK. (1981). "Organics vs. synthetics: Their use as additives in mortars." Simposio ICCROM - International Centre for the Study of the Preservation and Restoration of Cultural Property, Roma, 3 - 6 Noviembre, 25 - 52.

VALEK, J., HUGHES, J. J., GROOT, C. J. W. P(2012). "Historic mortars: characterisation, assessment and repair". Vol. 7 de RILEM Bookseries. New York London: Springer Netherlands. https://doi.org/10.1007/978-94-007-4635-0

VALERA, C. de (1869). La Santa Biblia: que contiene los sagrados libros del antiguo y nuevo testamento. Madrid: Depósito central de la Sociedad Bíblica.

WEBSTER, SUSAN V. (2011). "Vantage points: andeans and europeans in the construction of colonial Quito." Colonial latin american review 20 (3): 303-330. https://doi.org/10.1080/10609 164.2011.624330.

WEBSTER, S.V. (2012).“La desconocida historia de la construcción de la Iglesia de San Francisco." Procesos: Revista ecuatoriana de historia (35): 37-66.

\section{Autor/es}

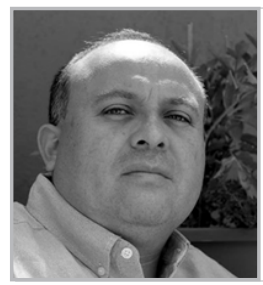

\section{Lenin Lara Calderón}

lenin.lara.calderon@alumnos.upm.es

Universidad Politécnica de Madrid

Arquitecto, con formación investigativa, docente y profesional, Máster en Restauración Arquitectónica y Máster Administración de Empresas Inmobiliarias, actualmente es estudiante de doctorado del Departamento de Construcción y Tecnología Arquitectónicas de la Universidad Politécnica de Madrid. Profesor investigador de la Universidad Internacional del Ecuador, ha colaborado en varios programas y proyectos de investigación sobre patología de la construcción y conservación del patrimonio

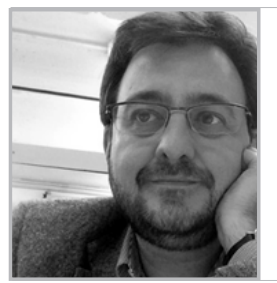

\section{David Sanz Arauz}

david.sanz.arauz@upm.es

Escuela Técnica Superior de Arquitectura de Madrid

Geólogo, PhD Geological Sciences, Máster en Restauración Arquitectónica. Desde 2000 es Profesor Asociado en el Departamento de Construcción y Tecnología Arquitectónicas de la Escuela Técnica Superior de Arquitectura de Madrid. En la actualidad es adjunto a la Subdirección de Doctorado, Postgrado e Investigación de la Escuela Técnica Superior de Arquitectura de Madrid

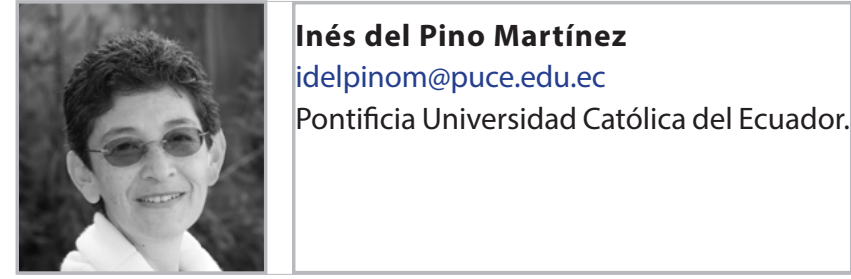

Arquitecta, Máster en Estudios de la Cultura por la Universidad Andina Simón Bolívar; Máster en Gobierno de la Ciudad con mención en Áreas Históricas; Doctora en Arte y Arquitectura por la Universidad Nacional de Colombia. Profesora investigadora de la Pontificia Universidad Católica del Ecuador. Investiga temas relacionados con territorio y paisaje, arquitectura y ciudad ecuatoriana
Artículo enviado el 05/09/2019 Artículo aceptado el 29/03/2020

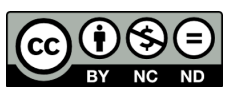

https://doi.org/10.37558/gec.v17i1.687 